\title{
Levodopa reversible loss of the Piper frequency oscillation component in Parkinson's disease
}

\author{
J H McAuley, D M Corcos, J C Rothwell, N P Quinn, C D Marsden
}

\begin{abstract}
Objectives-Although Parkinson's disease is typically characterised by bradykinesia, rigidity, and rest tremor, the possibility that two additional motor deficits are manifest during small hand muscle activity was explored-namely, weakness and abnormal physiological tremor.

Methods-A paradigm previously used in normal subjects reliably records the strength, tremor and surface EMG of index finger abducting contractions against a compliant (elastic) resistance. In addition to the well known physiological tremor at around $10 \mathrm{~Hz}$, there are other co existing peak tremor frequencies at around 20 and $40 \mathrm{~Hz}$; the last of these frequencies corresponds to the range of EMG Piper rhythm. The same technique was used to study parkinsonian patients while on and off dopaminergic medication.
\end{abstract}

Results-The maximum strength of finger abduction produced by first dorsal interosseous contraction was considerably lower when patients were off medication (mean (SD) $6.27(1.49) \mathrm{N}$ when off $v 12.33$ (3.64) $\mathrm{N}$ when on). There was also a marked reduction in the power of Piper frequency finger tremor $(p<0.0005)$ and EMG $(p<0.0005)$ oscillations that did not simply result from weaker contraction.

MRC Human

Movement and

Balance Unit, Institute

of Neurology, 23 Queen

Square, London

WC1N 3BG, UK

$\mathrm{J}$ H McAuley

J C Rothwell

C D Marsden (died 28

September 1998)

University

Department of Clinical

Neurology

N P Quinn

C D Marsden

School of Kinesiology and Department of Psychology, University of Illinois, Chicago, USA, and Department of Neurological

Sciences, Rush

Medical College,

Chicago, USA

D M Corcos

Correspondence to: Dr J H McAuley

jhmcauley@clara.co.uk

Received 16 December 1999 and in revised form

29 November 2000

Accepted 19 December 2000

strength of patients with idiopathic Parkinson's disease (IPD) is usually said to be normal, accurate quantitative measurement in large, particularly extensor, muscles has demonstrated weakness on isometric contraction. ${ }^{1-3}$ The basis for such weakness is uncertain but is likely to lie in abnormal descending inputs to the muscles resulting in inefficient or incomplete motor unit recruitment. Recently, it has been shown in the forearm extensor muscles of patients with IPD that weakness may result because a greater than normal proportion of motor units are trapped in a $10 \mathrm{~Hz}$ firing modulation during muscle contraction, preventing them from firing at higher frequencies and thereby generating stronger contractions. ${ }^{4}$ However, such firing abnormalities are not found in the triceps muscle, even though it displays the same degree of weakness in IPD. ${ }^{5}$ In addition to the trapping in a $10 \mathrm{~Hz}$ rhythm displayed by certain muscles in IPD, an additional and possibly more widespread abnormality could be loss of the higher frequency components of physiological tremor. Rumbling sounds at around $40 \mathrm{~Hz}$ may be heard in normal people on applying a stethoscope to a contracting muscle belly and such sounds seem to be absent during contractions made by parkinsonian patients (first noted by PA Merton (unpublished observations) and recorded subsequently). ${ }^{6}$ During compliant (elastic) finger muscle contraction in normal subjects, these rumbling sounds are shown to correspond to a quantifiable Piper frequency range oscillation component, a long described frequency modulation of EMG at $40-50 \mathrm{~Hz}^{7}$ As such modulations are thought to reflect central oscillations important in motor control, ${ }^{78}$ a similar technique used on patients with IPD may make it possible to quantify changes of peripherally manifest central oscillations in parkinsonism and to explore how they relate to the weakness and other deficits in this condition. Firstly, it was determined if weakness in IPD is a generalised phenomenon by seeing if it is present in the first dorsal interosseous muscle of the finger as well as in large proximal muscles. Secondly, the tremor and corresponding EMG oscillations of the finger were measured to determine if trapping of units into a slow $10 \mathrm{~Hz}$ rhythm may largely account for this weakness or if, as suggested by the negative studies on the triceps muscle, it is a finding peculiar to certain muscles such as the forearm extensors. Finally, as higher $40 \mathrm{~Hz}$ range rhythmic activity (and also intermediate $20 \mathrm{~Hz}$ range activity) can be accurately quantified using this finger muscle compliant contraction technique, an opportunity was afforded to test quantitatively the hypothesis that such oscillations are lost in IPD.
Patients and methods

In normal subjects the variability in the strength of peripheral oscillations, as measured by power spectra of tremor or EMG, is great and has been found to be considerably greater between subjects than between different recordings conducted on one subject. ${ }^{7}$ The variability present between different patients with neurological disease and of an older age range 
Table 1 Clinical details of patients studied. The maximum (worst possible) UPDRS motor score is 108 and is derived from subtotals for action tremor, rest tremor, rigidity, and bradykinesia

\begin{tabular}{llllll}
\hline Patient & Age $(y)$ & $\begin{array}{l}\text { Disease } \\
\text { duration }(y)\end{array}$ & UPDRS “On” & UPDRS “Off” & $\begin{array}{l}\text { UPDRS } \\
\text { difference }\end{array}$ \\
\hline 1 & 57 & 8 & 15 & 39 & 24 \\
2 & 51 & 11 & 14 & 43 & 29 \\
3 & 46 & 9 & 17 & 48 & 31 \\
4 & 51 & 4 & 12 & 44 & 32 \\
5 & 52 & 8 & 10 & 44 & 34 \\
\hline
\end{tabular}

UPDRS=Unified Parkinson's disease rating scale.

is likely to be even greater. With this in mind, a protocol was adopted in which oscillations were compared in the same patients when on and off medication. Five patients with IPD were selected for study on the basis that they fairly rapidly developed moderate to severe symptoms when taken off levodopa medication and recovered reliably and rapidly on reintroduction of their medication (table 1). As a result, all the patients could be studied when fully off and fully on medication in a single session. This meant that variability in clinical conditions from day to day and variability due to positioning of the recording electrodes and accelerometer could be eliminated. The patients were not on any medication other than levodopa. (Four of the patients had previously been tested for proximal muscle strength.) ${ }^{2}$ Informed consent was obtained from each patient and the study was conducted with local ethics committee approval. Oscillatory activity during steady abduction of the right index finger was recorded as previously described. ${ }^{7}$ The patient's right hand was placed palm downwards in an apparatus that held the hand and forearm steady while the index finger remained free to make a steady abducting contraction at the metacarpophalangeal joint against an elastic resistance. The force of contraction was measured by a strain gauge and could be preset by altering the stretch on the elastic. Tremor of the index finger was recorded by a miniature piezo resistive accelerometer (Vibro-Meter SA105, Fribourg, Switzerland) while surface EMG of first dorsal interosseous was recorded by two $9 \mathrm{~mm}$ diameter silver/silver chloride electrodes placed on the muscle belly and over the proximal phalanx. Surface EMG of the adducting palmar interosseous was recorded by an electrode between the second and third metacarpal muscles on the palmar aspect of the hand and one on the end of the middle finger. The DC accelerometer and strain gauge signals were amplified and digitised at $1000 \mathrm{~Hz}$ with 12 bit resolution by a 1401-plus (CED, Cambridge, UK) analogue to digital converter. The EMG signals were amplified by a Digitimer D (Welwyn, UK) amplifier and filtered with a low pass multistage filter (sixth order filter at around $300 \mathrm{~Hz}$ ) set to ensure that no frequencies would be present above the Nyqvist frequency for Fourier analysis. A high pass filter was set with a $3 \mathrm{~ms}$ time constant (53 $\mathrm{Hz}$ ) and applied to the EMG signal to prevent frequency artefacts due to electrode movement from appearing in these records, but preserving components resulting from fluctuations in amplitude of motor unit spikes. The EMG was digitally sampled and digitally full wave rectified. Such filtering and sampling have been shown to be suitable for Fourier analysis of EMG and detection of tremor related components. $^{7}$ All the signals were displayed and stored on computer disk by a software package (CED Spike 2) running on a PC microcomputer. A separate microcomputer ran a program which simultaneously triggered a recording period when the subject was ready and gave the subject a visual display indicating the start and end times of the recording period. Before trials measuring power spectra of tremor and EMG during compliant (elastic) contractions, each patient's maximum voluntary contraction (MVC) for isometric abduction of the index finger was assessed both when on and off medication by a non-compliant contraction against a strain gauge. The maximum force developed over the best of three 6 second contractions was recorded. Four patients were studied on first and off second while one was studied in the reverse order. Patients were noted to have widely different strengths when on and off. As contraction strength can influence the relative power of the peak oscillations at different frequencies, ${ }^{7}$ this had to be taken into consideration when comparing spectral power in the two conditions. (Maximum contraction was not recorded during every trial because this could not always be sustained for a 6 second recording period and because maximal contractions in normal subjects tend to reduce the strength of Piper rhythm frequency oscillations.) For a proper comparison of oscillations, on and off conditions were therefore compared (1) at the same contraction strength relative to maximum for that condition and (2) at the same absolute contraction strength. Thus, when studied on first, trials were conducted at $25 \%$ of the on MVC value. Then, when the patient came off, trials were conducted at $25 \%$ of off MVC (Off1 - same relative strength) and at a strength equal to the $25 \%$ "on" trial (Off-2-same absolute strength). In the single patient studied off first, trials were conducted at 25\% (Off- 1 ), $33 \%$, and $50 \%$ of off MVC, and then on coming on, at $25 \%$ of on MVC. The $50 \%$ off MVC proved to be similar in absolute strength to the $25 \%$ on MVC and so was selected as the Off-2 condition. At each contraction strength, 10 recordings each of 6.2 seconds duration were made. The digitised tremor and EMG data were analysed in the frequency domain by spectral analysis. The techniques used were similar to those previously employed. ${ }^{7}$ Finite fast Fourier transformation (FFT) with Hanning windowing was performed on each EMG trace using a commercial software package (CED Spike 2). The block size for each FFT was set to give a bin width of $1 \mathrm{~Hz}$ and data from 60 contiguous blocks were averaged to give the final overall power spectrum. The y axis of the spectral plots was root mean square power, equivalent to the variance of signal amplitude (a "square of signal amplitude" parameter). 


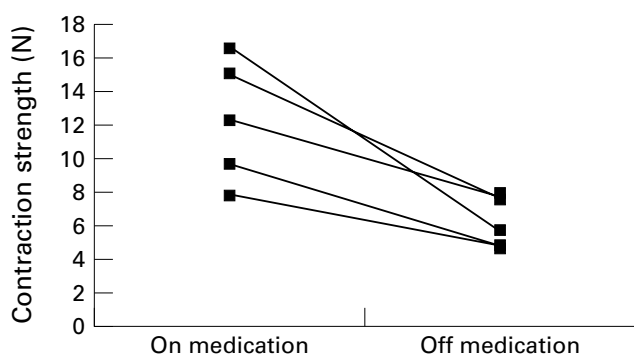

Figure 1 Maximum voluntary contraction strength (MVC) of abduction of the index finger in five patients with Parkinson's disease while on and off levodopa medication. The patients are weaker when off medication $(p=0.011$, paired $t$ test $)$.

\section{Results}

MAXIMUM CONTRACTION STRENGTH OF THE FIRST DORSAL INTEROSSEOUS

All patients were considerably weaker when off than when on medication (fig 1). The mean (SD) maximum force exerted in abduction by the end of the finger when off was 6.27 (1.49) $\mathrm{N}$ and when on it was 12.33 (3.64) N. This reduction to a mean of $52.8 \%$ of maximum strength when on was significant $(p=0.011$, paired $t$ test). EMG silence in the adducting small hand muscle demonstrated that weakness was not due to opposing antagonist activity.

TREMOR POWER SPECTRA DERIVED FROM ACCELEROMETER RECORDING

When the patients were on medication with few parkinsonian symptoms, averaged power spectral estimates of tremor (60 FFT blocks for each trial condition) showed the same three peaks in ranges around $10 \mathrm{~Hz}, 20 \mathrm{~Hz}$, and 40 $\mathrm{Hz}$ that were previously described in normal subjects (fig $2 \mathrm{~A}$ ). In one patient, a clear $40 \mathrm{~Hz}$ range peak was not apparent, although there was still considerable spectral power in this frequency range. When the patients were off medication and had moderate to severe parkinsonian symptoms, there were clear changes in the spectra (fig $2 \mathrm{~A}$ ). The power of the spectral peaks at $20 \mathrm{~Hz}$ tended to be reduced and $40 \mathrm{~Hz}$ Piper frequency peaks were absent. In the $40 \mathrm{~Hz}$ range, there was often not merely loss of the peak but very little power at all, so that the spectral distributions came to resemble those normally only seen at rest. ${ }^{7}$ This was elucidated by directly testing the resting state of the first dorsal interosseous muscle, recording with the finger simply gently extended against gravity instead of pulling against elastic force. There was no EMG activity in this muscle while in this posture. When off medication, there was often no clear difference in the spectral pattern (relative power at different frequencies) between resting and contracting conditions, although the overall spectral power was greater on contraction. Most of the patients reported having a parkinsonian-type $3-6 \mathrm{~Hz}$ rest tremor from time to time but, during these "resting" trials, tremor at this frequency was only apparent in one patient. Possibly, the high level of concentration during each 6 second trial period suppressed this tremor. Indeed, sometimes a slow rest tremor of the thumb and index finger was witnessed in the periods between trials. (Tremor at $3-6 \mathrm{~Hz}$ was not apparent in any patient during the forceful contractions that constituted the main part of this study.) By contrast with the higher frequency peaks, there was no reduction in 10 $\mathrm{Hz}$ range power, nor was there the consistent increase in power that had been previously reported in the forearm extensors. ${ }^{4}$ When brief periods were recorded of increased contraction strength of finger abduction from $50 \%$ MVC to near maximal (the strength level previously studied in the forearm extensors), there was still no clear increase in $10 \mathrm{~Hz}$ range power when the patients were off medication. There were no differences in the frequency values of any of the spectral peaks between the on and off conditions.

EMG POWER SPECTRA

Changes in EMG spectra generally paralleled those of tremor spectra (fig $2 \mathrm{~B}$ ). The loss of 40 $\mathrm{Hz}$ range power in the off condition was less apparent because of the greater background EMG power at high frequencies due to the spiky non-sinusoidal nature of the signal. Nevertheless, an indication of the lack of higher frequency EMG modulations was that the power in these ranges was often not distinguishable from the mean background level across the whole spectrum.

COHERENCE BETWEEN EMG AND TREMOR

SPECTRA

Coherence is a statistical measure of the similarity between two oscillatory signals so that if the signals carry oscillations of the same frequency with a constant phase relation, significant coherence will result at that frequency. Strong coherence was found in this study between tremor and corresponding EMG at all frequencies (fig $2 \mathrm{C}$ ), with peaks roughly in the 10,20 , and $40 \mathrm{~Hz}$ ranges, confirming that tremor and EMG record the same oscillatory processes. The significant coherence in the $40 \mathrm{~Hz}$ range when patients were off does not indicate that there was in fact a strong Piper rhythm frequency in such circumstances; a property of coherence is that its value reflects only the similarity between two signals and not their amplitudes. Thus a tiny strength oscillation present in two signals may be hidden by background noise in the power spectra yet be disclosed on coherence analysis. Phase plots, indicating the phase difference between coherent oscillations, had a reasonably constant slope at frequencies greater than $10 \mathrm{~Hz}$ (fig $2 \mathrm{D}$ ). This reflects the lag from EMG to tremor and is a measure of electromechanical coupling delay. ${ }^{7}$ In some patients, the slope tended to be more shallow when off, reflecting a shorter delay. Possibly, a more rigid hand resulted in faster coupling of fibre contraction to finger movement, but the data were insufficient to explore properly a correlation of coupling delay with clinical rigidity. 

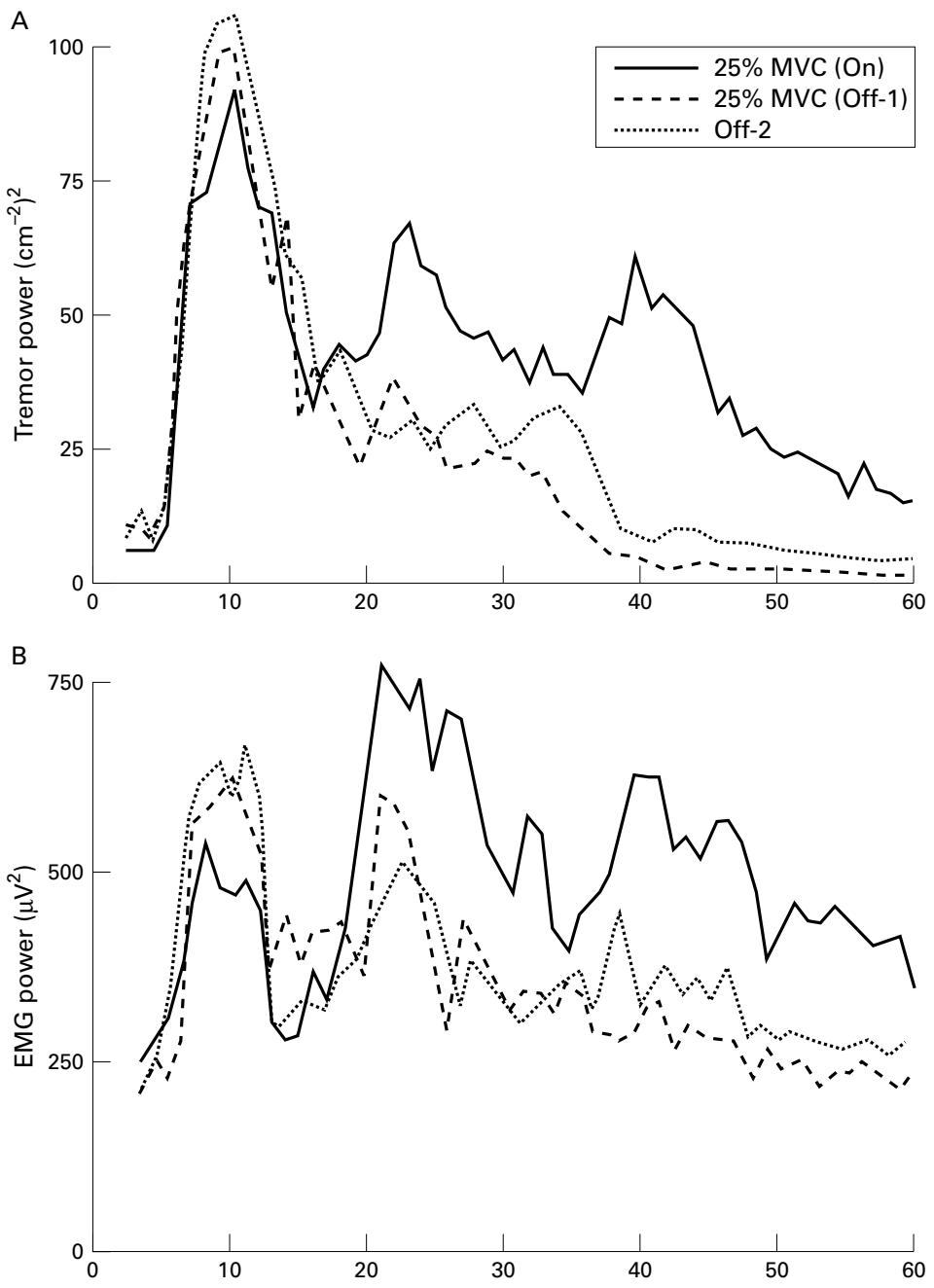

C
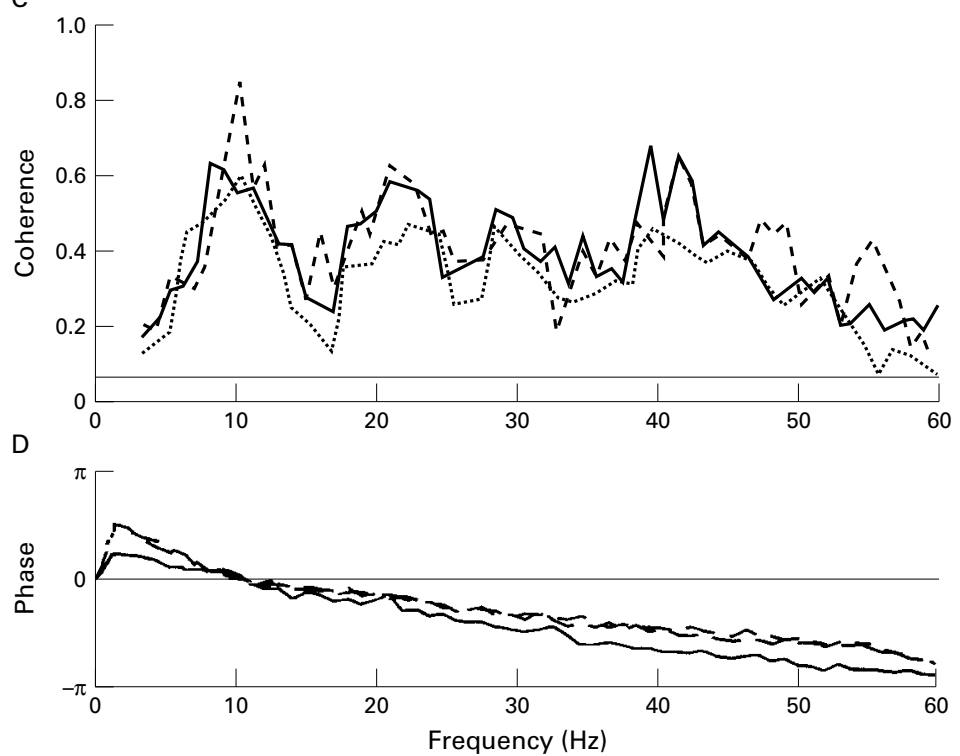

Figure 2. (A) Power spectra of finger tremor in a patient with IPD while on levodopa contracting at $25 \%$ maximum voluntary contraction (MVC) (solid line), while off medication at the same relative contraction strength (Off-1,25\% of off MVC) (dashed line) and while off medication at the same absolute contraction strength (Off-2,25\% of on MVC and around $50 \%$ of off $M V C$ in this patient) (dotted line). There is loss of $40 \mathrm{~Hz}$ range oscillatory activity when off, regardless of contraction strength, and a reduction of $20 \mathrm{~Hz}$ range activity. By contrast, the $10 \mathrm{~Hz}$ range peak is, if anything, somewhat larger. (B) Power spectra of $1 D I$ EMG during the same trial. There are similar reductions in $40 \mathrm{~Hz}$ range and $20 \mathrm{~Hz}$ range activities. (C) Coherence between tremor and EMG signals, showing significant coherence at all frequencies, which indicates that the two signals do indeed reflect the same oscillatory processes. The horizontal line marks the $95 \%$ confidence level for coherence being above that expected by chance. (D) The phase plot shows a lag of tremor behind EMG of around $12 \mathrm{~ms}$ when on and $6.5 \mathrm{~ms}$ when off (lag=slope of phaselfrequency).
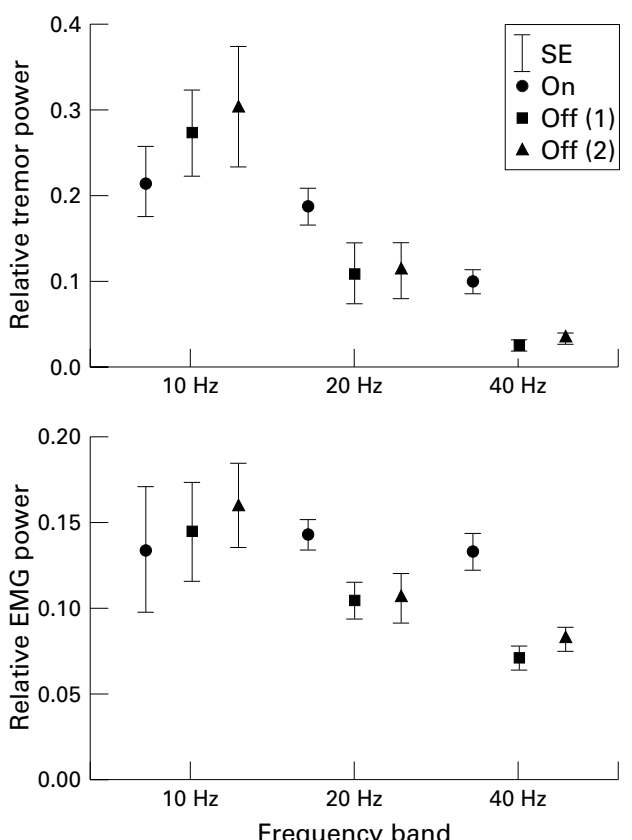

Figure 3 Mean power values of all the patients' frequency peaks in tremor and EMG spectra expressed relative to total spectral power from 2-50 $\mathrm{Hz}$. The solid bars represent trials while on medication contracting at $25 \% M V C(\mathrm{On})$, shaded bars represent trials off medication at the same relative contraction strength of $25 \%$ of off MVC (Off-1) and white bars represent trials off medication at the same absolute contraction strength (Off-2). The error bars indicate SEM. The asterisks below the $40 \mathrm{~Hz}$ frequencies indicate significant differences between the on and off conditions for tremor $(p=0.00011)$ and for $E M G$ $(p=0.00046)$.

QUANTIFICATION OF CHANGES IN SPECTRA

The above changes in tremor and EMG power spectra were quantified by determining the total power in a spectral peak and expressing this relative to the total power in the whole spectrum. Peak widths were set at four frequency bins (about $4 \mathrm{~Hz}$ ), being the value that best seemed to include peak bins while excluding background bins. The power over these bins was summed and then divided by the total power of all the bins from $2-50 \mathrm{~Hz}$. (The two lowest frequency bins were excluded from analysis because they contain artefact due to the Hanning window and gradual changes in baseline recording position.) When a peak was not apparent-for example, in the $20 \mathrm{~Hz}$ and $40 \mathrm{~Hz}$ ranges when off-a central value for the four bins was determined from the values of the corresponding peaks in other spectra of this patient. It should be noted that, using this method, a relative power of 0.083 indicates that the peak power is no greater than the overall mean power across the spectral range. Contraction strength normally has an effect on the relative power of the frequency peaks, often tending to increase high frequency power. Although this effect was not as great as the changes seen between the on and off parkinsonian states, it was important when making quantitative comparisons to correct for contraction strength because maximum contractions were weaker when off. However, it was not known whether the contraction effect would relate more to absolute force or to strength relative to maximum and so spectra 
when on were compared with those when off both at the same relative strength (Off-1) and at the same absolute strength (Off-2). The means of the patients' peak power values at each of the three frequency ranges were compared (fig 3). The $10 \mathrm{~Hz}$ range power tended to increase when off whereas the $20 \mathrm{~Hz}$ and $40 \mathrm{~Hz}$ range powers tended to decrease. This was apparent in both tremor and EMG spectra. On repeated measures analysis of variance (ANOVA), the drop in $40 \mathrm{~Hz}$ power was highly significant both for tremor $(\mathrm{p}<0.0005)$ and EMG ( $p<0.0005)$. Post hoc multiple comparisons using the Bonferroni correction (SPSS) indicated that the significant differences (at $p=0.05$ ) lay between On and Off-1 and between On and Off- 2 . At $20 \mathrm{~Hz}$, the drop was of doubtful significance (for tremor $p=0.16$; for EMG $p=0.050$; multiple comparisons NS). At $10 \mathrm{~Hz}$, the increase in peak power was only clearly reflected in two patients and was clearly not significant (for tremor, $p=0.56$; for $\mathrm{EMG}, \mathrm{p}=0.85$ ).

\section{Discussion}

The impaired maximal contraction strength (MVC) previously found in IPD in large proximal muscles ${ }^{1-3}$ is shown in this study also to involve a small hand muscle. This weakness is similarly levodopa reversible, as patients' strength when off medication roughly doubled when given their normal doses of levodopa. In the forearm extensors, parkinsonian weakness has been found to be accompanied by a clear increase in the power of $10 \mathrm{~Hz}$ range EMG oscillations and it has been suggested that trapping of motor units into this relatively slow firing rhythm could account for loss of strength. ${ }^{4}$ However, this does not seem to be a mechanism widely applicable to parkinsonian weakness because, as with the triceps muscle, ${ }^{5}$ there is only a slight and non-significant increase in $10 \mathrm{~Hz}$ oscillation in the finger when off medication despite a marked loss of strength. As near maximal contractions have been found in normal subjects to obscure the higher Piper range frequency peaks that were of interest in this study, only about $50 \%$ rather than near $100 \%$ MVC contractions were systematically measured. However, additional analysis of brief near maximal contractions while off medication still did not show a clear increase in 10 $\mathrm{Hz}$ oscillation power compared with similar contractions on medication. It has previously been shown that, using a system measuring compliant small hand muscle contractions, frequency analysis of tremor and EMG in normal subjects discloses three coexisting tremor oscillation frequency components, at around $10 \mathrm{~Hz}$, at around $20 \mathrm{~Hz}$, and at the Piper frequency range of $40 \mathrm{~Hz} .^{7}$ This study shows that when patients with IPD are on medication, these frequency peaks of oscillation are still present. By contrast, when the same patients are off medication, there is a dramatic and quantifiable loss of the $40 \mathrm{~Hz}$ Piper frequency oscillation component and a less convincing loss of the intermediate $20 \mathrm{~Hz}$ range component. The new tremor spectral distribution (not amplitude) comes to resemble the normal (and IPD) distribution while the muscle is inactive, even though, as in condition Off-2, the patient is contracting as strongly as when on medication. The loss of $40 \mathrm{~Hz}$ oscillation is in accord with previous studies on the forearm extensors that suggested an absence of Piper frequency vibrations on detecting muscle contractions of patients with IPD through a sound transducer. ${ }^{6}$ However, in these muscles, direct demonstration of the tremor and EMG correlates of the muscle sounds is difficult - clear 40 $\mathrm{Hz}$ range EMG peaks were not consistently found even in the on state or in normal people. Does this loss of the $40 \mathrm{~Hz}$ peak frequency of oscillation directly account for the weakness of small hand muscle contraction? Although the trapping into a $10 \mathrm{~Hz}$ rhythm is not a feature here, the loss of the $40 \mathrm{~Hz}$ peak could still indicate that units fire more slowly and so contract less strongly. However, this is unlikely for two reasons: (1) Both in patients on medication and in normal subjects, the power of the $20 \mathrm{~Hz}$ and $40 \mathrm{~Hz}$ peaks can be very variable between trials in the same subject but there is no such variability in subjects' strength. (2) It is unlikely that the $40 \mathrm{~Hz}$ Piper rhythm actually reflects units firing at that frequency. Instead it represents a frequency modulation of the unit population as a whole. ${ }^{9}{ }^{10}$ In other words, units firing at a range of frequencies from $10-30 \mathrm{~Hz}$ will all tend to be rather more likely to fire intermittently on beats of a pervasive $40 \mathrm{~Hz}$ rhythm than at other points in time. ${ }^{11}$ Even normal units cannot maintain firing at $40 \mathrm{~Hz}$ during a maximum contraction, yet the $40 \mathrm{~Hz}$ modulation clearly exists during contractions well below maximum. If, as in IPD, units lose this common modulation and so no longer fire together but at random timings with respect to one another, their oscillations will no longer summate and so will be poorly represented in power spectra, but there will still be the same summation of unit twitch forces, provided the individual units are firing as rapidly. To look at actual unit firing frequencies, single unit recordings are therefore required. Qualitative studies show that units are still able to fire rapidly when patients are off medication, ${ }^{12}$ indicating that tremor recordings do indeed mask the presence of rapidly but independently firing units. Weakness in IPD is likely to occur by a mechanism that results in a quantitative reduction in mean firing rate or unit twitch strength, regardless of the degree of common unit modulation, so that fewer units are firing at rapid rates than when on medication.

RELEVANCE OF THE LOSS OF HIGH FREQUENCY OSCILLATIONS

The high frequency modulatory activity recorded in these and previous studies is thought to represent the peripheral manifestation of rhythmic activity in the cerebral cortex; cortical oscillations modulate the firing of the motor unit population via descending pathways. ${ }^{8}$ For the reasons described above, it is unlikely that their absence in the periphery directly results in weakness through a limitation on the firing of individual units. Instead, it is possible that the loss of CNS oscillations generating the Piper 
rhythm frequency component could relate in a fundamental way to the levodopa reversible derangement of CNS motor control in IPD. Nevertheless, a loss of oscillations centrally might still involve aspects of motor control that influence key descending inputs which the motor neuron pool normally accesses for maximal contractions. In other words, the absence of the tremor and EMG oscillations does not directly cause parkinsonian weakness, but might still be a marker for the loss of certain inputs that do cause this weakness.

\section{Conclusions}

This study provides a clear demonstration of marked levodopa reversible small hand muscle weakness in IPD. In addition, quantitative analysis shows that normal Piper frequency (about $40 \mathrm{~Hz}$ ) components of tremor and EMG oscillations are lost in patients with IPD when medication is withdrawn. As the Piper rhythm is thought to be driven by cortical oscillatory activity, its loss may reflect that in IPD there is a change in the way voluntary commands access corticospinal outflow. One manifestation of this may be a failure to achieve normal maximum muscle contraction. Direct central recording of higher oscillation frequencies in IPD, rather than just the 3-6 Hz pathological rest tremor oscillation, could therefore yield useful information about the nature of different parkinsonian motor deficits and also provide clues about the role the oscillations may play in the normal brain.

JHMcA was supported by a Wellcome Research Training Fellowship grant.

1 Yanagawa S, Shindo M, Yanagisawa N. Muscular weakness in Parkinson's disease. Adv Neurol 1990;53:259-69.

2 Corcos DM, Chen C-M, Quinn NP, et al. Strength in Parkinson's disease: relation to rate of force generation and clinical status. Ann Neurol 1996;39:79-88.

3 Bridgewater KJ, Sharpe M. Trunk muscle performance in Bridgewater KJ, Sharpe M. Trunk muscle performance in
early Parkinson's disease. Physical Therapy 1998;78:56676.

4 Brown P, Corcos DM, Rothwell JC. Does parkinsonian action tremor contribute to muscle weakness in Parkinson's action tremor contribute to muscl

5 Brown P, Corcos DM, Rothwell JC. Action tremor and weakness in Parkinson's disease: a study of the elbow extensors. Mov Disord 1998;13:56-60.

6 Brown P. Muscle sounds in Parkinson's disease. Lancet 1997;349:533-5.

7 McAuley JH, Rothwell JC, Marsden CD. Frequency peaks of tremor, muscle vibration and electromyogram during human finger muscle contraction may reflect a rhythmicity of central neural firing. Exp Brain Res 1997;114:525-41.

8 Farmer SF. Rhythmicity, synchronization and binding in human and primate motor systems [review]. I Physiol 1998;509:3-14

9 Elble RJ, Randall JE. Motor unit activity responsible for 8-12 Hz component of human physiological finger tremor. 8-12 Hz component of human phys
f Neurophysiol $1976 ; 39: 370-83$.

10 Matthews PBC. The simple frequency response of human Matthews PBC. The simple frequency response of human
stretch reflexes in which either short or long latency components predominate. F Physiol 1994;481:777-98.

11 McAuley JH, Marsden CD. Physiological and pathological tremors and rhythmic central motor control [review]. Brain 2000;123;1545-67.

12 Young RR, Shahani BT. Single unit behaviour in human muscle afferent and efferent systems. Adv Neurol 1979;24: $175-83$.

\section{1st Asia Pacific Forum on Quality Improvement in Health Care}

\section{Three day conference}

\section{Wednesday 19 to Friday 21 September 2001 \\ Sydney, Australia}

We are delighted to announce this forthcoming conference in Sydney. Authors are invited to submit papers (call for papers closes on Friday 6 April), and delegate enquiries are welcome.

The themes of the Forum are:

- Improving patient safety

- Leadership for improvement

- Consumers driving change

- Building capacity for change: measurement, education and human resources

- The context: incentives and barriers for change

- Improving health systems

- The evidence and scientific basis for quality improvement.

Presented to you by the BMJ Publishing Group (London, UK) and Institute for Healthcare Improvement (Boston, USA), with the support of the the Commonweatlh Department of Health and Aged Care (Australia), Safety and Quality Council (Australia), NSW Health (Australia) and Ministry of Health (New Zealand).

For more information contact: quality@bma.org.uk or fax +44 (0) 73836869 